\title{
Analysis and Numerical Approximation of a Free Boundary Problem for a Singular Ordinary Differential Equation
}

P. LIMA ${ }^{1}$, Departamento de Matemática, Instituto Superior Técnico, Av. Rovisco Pais, 1049-001 Lisboa, Portugal

L. MORGADO ${ }^{2}$, Departamento de Matemática, Universidade de Trás-os-Montes e Alto Douro, Quinta de Prados, 5001-801 Vila Real, Portugal.

\section{Introduction}

Abstract. We analyse a free boundary problem for a second order nonlinear ordinary differential equation. The asymptotic behavior of the solutions satisfying certain boundary conditions is analysed at the endpoints of the interval where the solution is sought. Based on this study, an efficient shooting method is introduced and numerical results are obtained.

In this paper we consider the following equation

$$
y^{\prime \prime}+g(x) y^{\prime}+f(y)=0, \quad 0<x<+\infty,
$$

where we assume that

$$
\begin{gathered}
g(x)=\frac{N-1}{x}, \quad N \geq 2, \\
f(y)=a y^{q}-b y^{p}, \quad 0 \leq p<q \leq 1, \quad a, b>0 .
\end{gathered}
$$

We look for a finite point $M>0$ and a solution $y$ satisfying

$$
y(x)>0, \quad 0<x<M, \quad y^{\prime}(0)=0, \quad y(M)=y^{\prime}(M)=0,
$$

This kind of problems arise when we are looking for radial solutions of the elliptic equation $\triangle y+f(y)=0$ in a ball $B(0, M) \subset \mathbb{R}^{N}$, and has several applications in physics.

Recently, the following model was proposed in plasma physics, for Tokamac equilibria with magnetic islands [6]:

$$
\begin{array}{rlrl}
\frac{1}{x}\left(x G^{\prime}\right)^{\prime} & =\gamma+\alpha\left(G_{s}-G\right)^{\frac{1}{2}}, & & 0<x<x_{s} \\
G^{\prime}(0) & =0, \quad G\left(x_{s}\right)=G_{s}, & G^{\prime}\left(x_{s}\right)=0
\end{array}
$$

\footnotetext{
${ }^{1}$ plima@math.ist.utl.pt

${ }^{2}$ luisam@utad.pt
} 
where $\gamma=-0.2066, \alpha=5.9$ and $\beta=-2.5$, and constitutes the motivation for the present work.

Performing the variable substitution $y=G_{s}-G$, problem (1.5)-(1.6) becomes a particular case of the singular free boundary value problem (1.1)-(1.4), with $p=0$, $q=\frac{1}{2}, a=\alpha, b=-\gamma$ and $N=2$.

The questions of existence and uniqueness of a finite point $M>0$ and a positive solution of problem (1.1)-(1.4), were studied, for example, by Kaper and Kwong, [2]. These authors stated the following

Theorem 1.1. If in equation (1.1)

(H1) $f$ is continuous on $[0, \infty)$ and locally Lipschitz on $(0, \infty)$;

(H2) there exists a $\beta_{0}>0$ such that $F(y)>0$ for all $0<y<\beta_{0}, F\left(\beta_{0}\right)=0$, and $f(y)>0$ for $y \geq \beta_{0}$, where $F(y)=-\int_{0}^{y} f(s) d s, \quad y>0$;

(H3) $\int_{0}^{\beta_{0}} F^{-\frac{1}{2}}(y) d y<\infty$;

(H4) $\liminf _{y \rightarrow \infty} f(y)>0$;

(H5) $y \mapsto \frac{f(y)}{y-\beta_{0}}$ is nonincreasing for $y>\beta_{0}$;

(H6) $g(x) \geq 0$ for all $x \geq 0$;

(H7) $\lim _{x \rightarrow \infty} g(x)=0$;

(H8) $g$ is continuous on $(0, \infty)$ and $g(x)=O\left(x^{-1}\right)$ as $x \rightarrow 0$;

(H9) $g$ is monotone nonincreasing;

(H10) $x \mapsto g(x) \exp \left(2 \int g(x) d x\right)$ is nondecreasing;

then problem (1.1)-(1.4) has one and only one solution.

When $f$ and $g$ in equation (1.1), are given by (1.2) and (1.3), conditions (H1), (H4), (H6), (H7), (H8), (H9) and (H10) are automatically satisfied. Condition (H2) is satisfied with $\beta_{0}=\left(\frac{b(q+1)}{a(p+1)}\right)^{\frac{1}{q-p}}$. The integral in condition (H3) will be convergent if $p<1$. Finally, condition (H5) holds iff $\frac{d}{d y}\left(\frac{f(y)}{y-\beta_{0}}\right) \leq 0, \forall y>\beta_{0}$, what is equivalent to say that

$$
h(y)=\left(a q y^{q-1}-b p y^{p-1}\right)\left(y-\beta_{0}\right)-\left(a y^{q}-b y^{p}\right) \leq 0, \quad \forall y>\beta_{0} .
$$

When $q<1, h$ attains a maximum at $\beta_{M}=\left(\frac{b p(p-1)}{a q(q-1)}\right)^{\frac{1}{q-p}}$. If $\beta_{0} \geq \beta_{M}$, which holds when $0 \leq p<q \leq \frac{1}{\sqrt{3}}$ or $\frac{1}{\sqrt{3}}<q<1$ and $0 \leq p<-\frac{q}{2}+\frac{1}{2} \sqrt{4-3 q^{2}}$, (1.7) is satisfied since $h\left(\beta_{0}\right) \leq 0$ and $h(y)<h\left(\beta_{0}\right)$, for every $y>\beta_{0}$. If $\beta_{M}>\beta_{0}$, what happens when $\frac{1}{\sqrt{3}}<q<1,-\frac{q}{2}+\frac{1}{2} \sqrt{4-3 q^{2}} \leq p<q$, (1.7) will be satisfied if $h\left(\beta_{M}\right)<0$. It can be easily proved that this last condition holds if $\frac{(q-1)(p-1)}{p q}-\left(\frac{q^{3}-q}{p^{3}-p}\right)^{\frac{1}{q-p}}<0$. 
Finally, if $q=1$ and $p \neq 0$, since $f(y)>0$ for $y \geq \beta_{0}$, we have

$$
h(y)>\left(a-b p y^{p-1}\right)\left(y-\beta_{0}\right), \quad \forall y \geq \beta_{0} .
$$

The point $y^{*}=\left(\frac{b(q+1)}{a p}\right)^{\frac{1}{q-p}}>\beta_{0}$ satisfies $h\left(y^{*}\right)>a\left(1-\frac{p^{2}}{2}\right)\left(y^{*}-\beta_{0}\right)>0$, $\forall 0<p<1$, so (1.7) will not be satisfied if $q=1$ and $p \neq 0$. When $p=0$ and $q=1,(1.7)$ holds since $h(y)=-b$. Resuming,

Corollary 1.1. If in equation (1.1) $f$ and $g$ are defined by (1.2) and (1.3) and if one of the following conditions is satisfied

(i) $0 \leq p<q \leq \frac{1}{\sqrt{3}}$;

(ii) $\frac{1}{\sqrt{3}}<q<1$ and $0 \leq p \leq-\frac{q}{2}+\frac{1}{2} \sqrt{4-3 q^{2}}$;

(iii) $\frac{1}{\sqrt{3}}<q<1,-\frac{q}{2}+\frac{1}{2} \sqrt{4-3 q^{2}}<p<q$ and $\frac{(q-1)(p-1)}{p q}-\left(\frac{q^{3}-q}{p^{3}-p}\right)^{\frac{1}{q-p}} \leq 0$;

(iv) $p=0$ and $q=1$;

then problem (1.1)-(1.4) has one and only one solution.

In order to obtain approximate solutions of the free boundary value problem (1.1)-(1.4), singular at $x=0$, we will extend the analytical-numerical approach used in [5] and [4]. In particular, we will determine a one-parameter family of solutions of equation (1.1) that satisfy the boundary condition at $x=0$, and then we introduce an iterative method which enable us to determine, simultaneously, the value of the parameter of that family and the value of $M$, in such a way that the boundary conditions at $x=M$ are also satisfied.

\section{Behavior of the Solutions in the Neighborhood of the Singular Point $x=0$}

Consider the singular Cauchy problem

$$
\begin{aligned}
& y^{\prime \prime}+\frac{N-1}{x} y^{\prime}+a y^{q}-b y^{p}=0, \quad 0<x<+\infty, \\
& y(0)=y_{0}, \quad y^{\prime}(0)=0 .
\end{aligned}
$$

The questions of existence and uniqueness of solutions of singular Cauchy problems of this kind were studied by Konyukhova [3], where asymptotic expansions of the solution in the neighborhood of singular points were also presented. In order to apply those results, we perform the variable substitution

$$
\omega_{1}=y-y_{0}, \quad \omega_{2}=x y^{\prime} .
$$

(2.1), (2.2) can be rewritten as

$$
\begin{aligned}
& x \omega^{\prime}=A \omega+H\left(x, \omega, y_{0}\right)+G\left(x, y_{0}\right) \\
& \omega\left(x, y_{0}\right)=0,
\end{aligned}
$$


where $\omega=\left(\omega_{1}, \omega_{2}\right)^{t}, H=\left(H_{1}, H_{2}\right)^{t}, G=\left(G_{1}, G_{2}\right)^{t}, H_{1}=G_{1}=0, H_{2}=$ $-a x^{2}\left(\omega_{1}+y_{0}\right)^{q}+b x^{2}\left(\omega_{1}+y_{0}\right)^{p}+a x^{2} y_{0}^{q}-b x^{2} y_{0}^{p}, G_{2}=-a x^{2} y_{0}^{q}+b x^{2} y_{0}^{p}$ and $A=$ $\left[\begin{array}{cc}0 & 1 \\ 0 & 2-N\end{array}\right]$, whose eigenvalues are $\lambda_{1}=0$ and $\lambda_{2}=2-N \leq 0, \forall N \geq 2$.

As it can be easily seen, we are in conditions to use Theorem 5 of [3] to conclude that for any fixed positive $y_{0}$ and any $N \geq 2$, problem (2.1), (2.2) has (for sufficiently small $x$ ) a unique solution, $y\left(x, y_{0}\right)$, holomorphic at $x=0$, and that may be represented by

$$
y\left(x, y_{0}\right)=y_{0}+\sum_{k=1}^{+\infty} y_{2 k}\left(y_{0}\right) x^{2 k}, \quad 0 \leq x \leq \delta, \quad \delta>0,
$$

where the coefficients $y_{2 k}$ may be determined substituting (2.3) in (2.1). The first of these coefficients are

$$
\begin{aligned}
& y_{2}=\frac{b y_{0}^{p}-a y_{0}^{q}}{2 N}, y_{4}=\frac{b p y_{0}^{p}-a q y_{0}^{q}}{4(2+N) y_{0}} y_{2} \\
& y_{6}=\frac{b p y_{0}^{p}\left((p-1)^{2} y_{2}^{2}+2 y_{0} y_{4}\right)-a q y_{0}^{q}\left((q-1)^{2} y_{2}^{2}+2 y_{0} y_{4}\right)}{12(4+N) y_{0}^{2}} .
\end{aligned}
$$

We have just proved the following

Proposition 2.1. For any $N \geq 2$ and $y_{0}>0$, problem (2.1), (2.2) has a unique solution which is holomorphic in the neighborhood of $x=0$ and may be represented in the form of the series (2.3). The first coefficients of this series are given by (2.4).

\section{Behavior of the Solutions in the Neighborhood of $x=M$}

Consider now the Cauchy problem

$$
\begin{aligned}
& y^{\prime \prime}+\frac{N-1}{x} y^{\prime}+a y^{q}-b y^{p}=0, \\
& y(M)=y^{\prime}(M)=0,
\end{aligned}
$$

where $M$ is a fixed positive number. Since $q>p \geq 0$, from the conditions (3.2) it follows that any solution $y$ of this problem satisfies:

$$
\lim _{x \rightarrow M^{-}} \frac{y(x)}{(M-x)^{2}}=C<\infty .
$$

If $p=0$ then $C=b / 2>0$ and $y(x)=b / 2(M-x)^{2}+o\left((M-x)^{2}\right)$ as $x \rightarrow M^{-}$. When $p>0$, we have $C=0$ and it is obvious that the function $y(x) \equiv 0$ satisfies (3.1), (3.2). However, it is possible to verify that for each $M$ this problem has also a positive solution, which can be the solution of our free boundary problem. Let us 
obtain the main term of the series expansion of this solution. Let us assume that, for a certain positive $k$, we have

$$
y(x)=C(M-x)^{k}(1+o(1)) ;
$$

then

$$
\begin{aligned}
& y^{\prime}(x)=C k(M-x)^{k-1}(1+o(1)) \\
& y^{\prime \prime}(x)=C k(k-1)(M-x)^{k-2}(1+o(1)) .
\end{aligned}
$$

Substituting (3.3), (3.4), (3.5) in equation (3.1) and taking into account (3.2), we conclude that

$$
\lim _{x \rightarrow M_{-}} \frac{y^{\prime \prime}(x)+(N-1) / x y^{\prime}(x)+a y(x)^{q}}{y(x)^{p}}=\lim _{x \rightarrow M-} \frac{C k(k-1)(M-x)^{k-2}+o\left((M-x)^{k-2}\right)}{C^{p}(M-x)^{k p}+o\left((M-x)^{k-2}\right)}=b,
$$

from where it follows that $k=2 /(1-p), C=\left(\frac{b(1-p)^{2}}{2(1+p)}\right)^{1 /(1-p)}$. Hence, for $p>0$, in the neighborhood of $x=M$, we have

$$
y(x)=\left(\frac{b(1-p)^{2}}{2(1+p)}\right)^{1 /(1-p)}(M-x)^{2 /(1-p)}(1+o(1)) .
$$

Remark: Note that since $q<1, f(y)$ is not lipschitzian when $y=0$ and this explains the multiplicity of solutions of the Cauchy problem (3.1),(3.2).

\section{Parameter Estimates}

According to lemma 1.2.1 in [1], any solution $y(x)$ of (1.1),(1.4) satisfies $F(y(0))>$ 0 , therefore

$$
y_{0}=y(0)>\beta_{0}=\left(\frac{b(q+1)}{a(p+1)}\right)^{\frac{1}{q-p}} .
$$

Let us consider the family of functions

$$
\bar{y}\left(x, y_{0}\right)=y_{0}+\frac{b y_{0}^{p}-a y_{0}^{q}}{2 N} x^{2}, \quad y_{0}>0 .
$$

Each one of these functions vanishes at

$$
\tilde{M}\left(y_{0}\right)=\sqrt{\frac{2 N}{a y_{0}^{q-1}-b y_{0}^{p-1}}} .
$$

Let us minimize (4.3) taking (4.1) into account. When $0 \leq p<q<1, \tilde{M}\left(y_{0}\right)$ attains a minimum at the point $y_{0}=\left(\frac{b(p-1)}{a(q-1)}\right)^{\frac{1}{q-p}}$, and in this case $\min _{y_{0}>\beta_{0}} \tilde{M}\left(y_{0}\right)=$ 
$\sqrt{2 N\left(\frac{b(p-1)}{a(q-1)}\right)^{\frac{1-p}{q-p}} \frac{1-q}{b(q-p)}}$. When $q=1$ and $p=0, \tilde{M}\left(y_{0}\right)$ is strictly decreasing for all $y_{0}>\beta_{0}$, so $\inf _{y_{0}>\beta_{0}} \tilde{M}\left(y_{0}\right)=\lim _{y_{0} \rightarrow+\infty} \tilde{M}\left(y_{0}\right)=\sqrt{2 N / a}$. Define

$$
M_{\min }= \begin{cases}\sqrt{2 N\left(\frac{b(p-1)}{a(q-1)}\right)^{\frac{1-p}{q-p}} \frac{1-q}{b(q-p)}}, & \text { if } \quad 0 \leq p<q<1 \\ \sqrt{2 N / a}, & \text { if } \quad q=1, p=0 .\end{cases}
$$

We shall now show that this value is actually a lower bound of $M$. With this purpose we shall begin by deducing some auxiliary results.

Lemma 4.1. Let $y\left(x, y_{0}\right)$ be a solution of the Cauchy problem (2.1),(2.2), with $y_{0}>\beta_{0}$. If $\hat{x}$ is the least positive value of $x$, such that $y^{\prime}\left(\hat{x}, y_{0}\right)=0$, then

1. there exists $x_{1}<\hat{x}$, such that $y\left(x_{1}, y_{0}\right)=u_{0}$, where $u_{0}$ is the positive root of $f$;

2. there exists $x_{2}<x_{1}$, such that $y^{\prime \prime}\left(x_{2}, y_{0}\right)=0$.

Proof. First of all, let us remark that since $\hat{x}$ is a point of local minimum of $y$, then $y\left(\hat{x}, y_{0}\right)<u_{0}$, where $u_{0}$ is the positive root of $f(y)$. Actually, from equation (2.1) it follows that $y^{\prime \prime}\left(\hat{x}, y_{0}\right)=-f\left(y\left(\hat{x}, y_{0}\right)\right) \geq 0$ and therefore $y\left(\hat{x}, y_{0}\right) \leq u_{0}$. Moreover, we must have $y\left(\hat{x}, y_{0}\right) \neq u_{0}$ (otherwise we would have $y^{\prime \prime}\left(\hat{x}, y_{0}\right)=y^{\prime}\left(\hat{x}, y_{0}\right)=0$ and $y$ would be constant: $\left.y\left(x, y_{0}\right) \equiv u_{0}\right)$. Hence we have $y\left(x_{1}, y_{0}\right)=u_{0}$, for a certain $x_{1}<\hat{x}$. This proves the first part of the lemma. Now, since $y^{\prime}\left(x_{1}, y_{0}\right)<0$ and $f\left(y\left(x_{1}, y_{0}\right)\right)=0$, from equation (2.1) it follows that $y^{\prime \prime}\left(x_{1}, y_{0}\right)>0$. From the continuity of $y^{\prime \prime}$ we conclude that there exists $x_{2}<x_{1}$ such that $y^{\prime \prime}\left(x_{2}, y_{0}\right)=0$.

Lemma 4.2. Let $y\left(x, y_{0}\right)$ be a solution of the Cauchy problem (2.1),(2.2), with $y_{0}>\beta_{0}$, and such that $y^{\prime}\left(\hat{x}, y_{0}\right)=0$, for some $\hat{x}>0$. Then $y\left(x, y_{0}\right)>\bar{y}\left(x, y_{0}\right)$, for $0<x \leq \hat{x}$, where $\bar{y}$ is given by (4.2).

Proof. From formula (2.3) it follows that $y^{\prime \prime}\left(0, y_{0}\right)=\bar{y}^{\prime \prime}\left(0, y_{0}\right)=2 y_{2}(y 0)<0$. Moreover, from formulae $(2.4)$, we have $y^{\prime \prime \prime}\left(0, y_{0}\right)=0$ and $y^{(4)}\left(x, y_{0}\right)>0$ in the neighborhood of $x=0$, and therefore $y^{\prime \prime}\left(x, y_{0}\right)$ is increasing with respect to $x$. By lemma 4 , there exists $x_{2}<\hat{x}$, such that $y^{\prime \prime}\left(x_{2}, y_{0}\right)=0$, that is, $y^{\prime}\left(x, y_{0}\right)$ has a local minimum at $x=x_{2}$. Moreover, $y^{\prime \prime}\left(x, y_{0}\right)>0$, and therefore $y^{\prime}$ is increasing, for $x_{2}<x<\hat{x}$, because $y\left(x, y_{0}\right)<u_{0}$, for such values of $x$. Hence we have

$$
y^{\prime}\left(x, y_{0}\right)>\bar{y}^{\prime}\left(x, y_{0}\right)=2 y_{2}\left(y_{0}\right) x
$$

for $0<x<\hat{x}$, and

$$
y\left(x, y_{0}\right)=y_{0}+\int_{0}^{x} y^{\prime}\left(t, y_{0}\right) d t>y_{0}+\int_{0}^{x} \bar{y}^{\prime}\left(t, y_{0}\right) d t=\bar{y}\left(x, y_{0}\right),
$$

for $0<x \leq \hat{x}$.

Finally, from lemma 4 and lemma 5, we obtain the needed result. 
Corollary 4.2. If $M$ and $\bar{y}_{0}$ are such that $y\left(M, \bar{y}_{0}\right)=y^{\prime}\left(M, \bar{y}_{0}\right)=0$, where $y$ is a solution of the Cauchy problem (2.1), (2.2), then $M>M_{\min }$.

Proof. Since $\bar{y}_{0} \geq \beta_{0}$ and $y\left(x, \bar{y}_{0}\right)$ has a local minimum at some $M, y\left(x, \bar{y}_{0}\right)$ satisfies the conditions of lemma 5 , with $M=\hat{x}$. Therefore we can assure that $y\left(x, \bar{y}_{0}\right)>$ $\bar{y}\left(x, \bar{y}_{0}\right)$, for $0<x \leq M$. In particular, since $\bar{y}\left(\tilde{M}\left(\bar{y}_{0}\right), \bar{y}_{0}\right)=0$, by construction, we conclude that

$$
M>\tilde{M}\left(\bar{y}_{0}\right) \geq M_{m i n},
$$

which concludes the proof of the corollary.

\section{Numerical Results}

In order to compute a numerical solution of the free boundary problem (1.1)-(1.4) we consider the Cauchy problems (2.1)-(2.2) and (3.1)-(3.2) separately.

The numerical solution of the singular CP (2.1)-(2.2) is obtained by replacing the boundary conditions (2.2) by

$$
\begin{gathered}
y(\delta)=y_{0}+y_{2}\left(y_{0}\right) \delta^{2}+y_{4}\left(y_{0}\right) \delta^{4}+y_{6}\left(y_{0}\right) \delta^{6} \\
y^{\prime}(\delta)=2 y_{2}\left(y_{0}\right) \delta+4 y_{4}\left(y_{0}\right) \delta^{3}+6 y_{6}\left(y_{0}\right) \delta^{5}
\end{gathered}
$$

where $\delta$ is sufficiently close to 0 and $y_{0}$ is a fixed positive number. The functions $y_{2}, y_{4}$ and $y_{6}$ are defined by (2.4). According to Proposition 2.1, if $\delta$ is close to 0 , the right-hand side of the first condition (5.1) gives an accurate representation of the exact solution of (2.1)-(2.2).

In our computations, we have used the NDSolve subroutine of Mathematica to solve the regular Cauchy problem (2.1),(5.1). Some numerical solutions obtained by this method, for a particular case of problem (2.1)-(2.2), are displayed in fig.1-a).

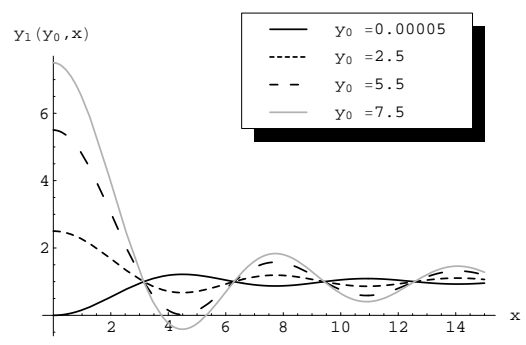

a)

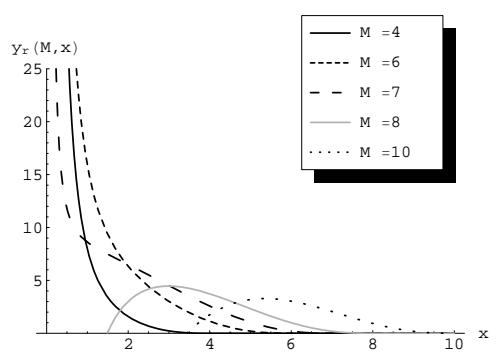

b)

Figure 1: Solution of problem a) (2.1), (2.2) for different values of $y_{0}$, with $a=b=1$, $p=0, q=1$ and $N=3 ; \mathrm{b})(3.1),(3.2)$ for different values of $M$, with $a=b=1$, $p=0.2, q=0.8$ and $N=4$.

On the other hand, the approximate solution of the Cauchy problem near $x=$ $M$ can be obtained in different ways. When $p=0$, as we have seen in Section 3 , the problem has a unique solution for each $M>0$, which can be computed straightforward using again the NDSolve routine. In the case $p>0$ the use of 
NDSolve (or any other standard routine for Cauchy problems) will produce the trival solution $y \equiv 0$ which is not the solution of our problem. In order to obtain the needed solution, we need to use the information about its asymptotics, obtained in Section 3. Namely, we must replace the boundary conditions (3.2) by

$$
\begin{gathered}
y(M-\epsilon)=w(M-\epsilon), \\
y^{\prime}(M-\epsilon)=w^{\prime}(M-\epsilon),
\end{gathered}
$$

where $\epsilon$ is sufficiently close to 0 and $w(x)$ is the main term of the series expansion (3.6). Then we use NDSolve to compute the Cauchy problem (3.1),(5.2),(5.3). Figure 1-b) shows the graphics of the solutions computed for a particular case of the Cauchy problem (3.1)-(3.2), using this method.

Let us now denote by $y_{l}\left(y_{0}, x\right)$ the solution of equation (1.1) which satisfies the boundary conditions (5.1), for a certain $y_{0}$. By $y_{r}(M, x)$ we shall denote the solution of equation (1.1) which satisfies the condition (3.2) (if $p=0$ ) or (5.2),(5.3) (if $p>0$ ), for a certain $M$. We shall look for the solution of the free boundary problem in the form

$$
y(x)= \begin{cases}y_{l}\left(y_{0}, x\right), & \delta \leq x \leq M / 2 \\ y_{r}(M, x), & M / 2 \leq x \leq M-\epsilon\end{cases}
$$

By construction, a function of this form will be a solution of the free boundary problem (1.1)-(1.4) if and only if the following conditions are satisfied

$$
y_{l}\left(y_{0}, M / 2\right)=y_{r}(M, M / 2) ; y_{l}^{\prime}\left(y_{0}, M / 2\right)=y_{r}^{\prime}(M, M / 2) .
$$

The conditions (5.4) form a nonlinear set of two equations with respect to the unknowns $y_{0}$ and $M$, which can be efficiently solved, for example, by the Newton's method.

In our computations we have used $\delta=0.001, \epsilon=0.001$ and as initial values for $M$ and $y_{0}$ we used the estimates obtained in Section 4. The stopping condition for the Newton's method was

$$
\max \left\{\left|y_{l}\left(y_{0}, M / 2\right)-y_{r}(M, M / 2)\right|,\left|y_{l}^{\prime}\left(y_{0}, M / 2\right)-y_{r}^{\prime}(M, M / 2)\right|\right\}<10^{-15}
$$

Under these conditions, the Newton's method converged after 9 iterations (in average).

To illustrate our numerical results, we compute the solution of problem (1.1)(1.4) for several cases corresponding to different values of $a, b, p, q$ and $N$. The approximate values of $M$ and $y_{0}, \bar{M}$ and $\overline{y_{0}}$, respectively, are displayed in tables 1 and 2 . In figure 2 , we plot the corresponding solutions.

Note that when $q=1, p=0$, and for example $N=2$, the general solution of equation (2.1) is given by the exact formula $y(x)=\frac{b}{a}+C J_{0}(\sqrt{a} x)$, where $J_{0}$ is the Bessel function, and $C$ is a constant. Taking into account the boundary conditions at $x=M$, we obtain the values of $M$ and $C$, and therefore the value $y_{0}=y(0)$, for some given values of $a$ and $b$. In table 3 we compare these values with those obtained by our numerical algorithm. From this comparison we conclude that all the obtained approximations of $y_{0}$ and $M$ have eight digits of accuracy. 


\begin{tabular}{|c|c|c|c|c|}
\hline$q$ & $\overline{y_{0}}$ & $\beta_{0}$ & $\bar{M}$ & $M_{\min }$ \\
\hline 0.3 & 6.04623 & 2.19112 & 22.7135 & 15.3767 \\
0.4 & 5.86222 & 2.12873 & 12.9553 & 8.5582 \\
0.6 & 5.56924 & 2.02448 & 8.31109 & 5.13538 \\
0.7 & 5.44974 & 1.98040 & 7.26542 & 4.29187 \\
\hline
\end{tabular}

Table 1: $a=b=1, p=0.25, N=3$

\begin{tabular}{|c|c|c|c|c|}
\hline$p$ & $\overline{y_{0}}$ & $\beta_{0}$ & $\bar{M}$ & $M_{\min }$ \\
\hline 0.0 & 8.64506 & 2.08493 & 6.09792 & 3.86697 \\
0.2 & 8.14901 & 1.96556 & 7.07127 & 4.11489 \\
0.3 & 7.96416 & 1.91716 & 7.82395 & 4.29955 \\
0.4 & 7.81274 & 1.87440 & 8.89730 & 4.55901 \\
\hline
\end{tabular}

Table 2: $a=b=1, q=0.8, N=4$

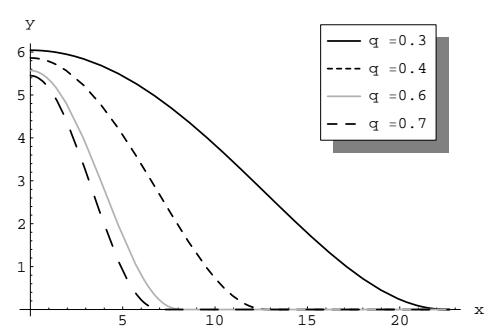

a)

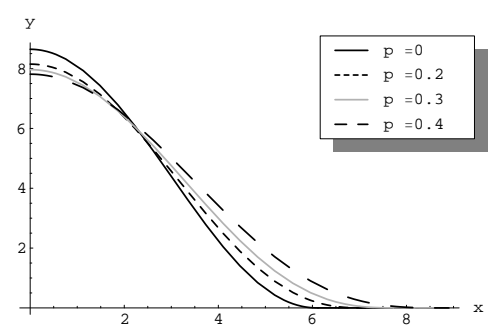

b)

Figure 2: a) $a=b=1, p=0.25, N=3$; b) $a=b=1, q=0.8, N=4$.

\begin{tabular}{|c|c|c|c|c|}
\hline$a, b$ & $y_{0}$ & $\overline{y_{0}}$ & $M$ & $\bar{M}$ \\
\hline 1,1 & $\mathbf{3 . 4 8 2 8 7 1 9 4}$ & $\mathbf{3 . 4 8 2 8 7 1 9 7}$ & $\mathbf{3 . 8 3 1 7 0 5 9 7}$ & $\mathbf{3 . 8 3 1 7 0 5 9 9}$ \\
1,2 & $\mathbf{6 . 9 6 5 7 4 3} 87$ & $\mathbf{6 . 9 6 5 7 4 3 7 8}$ & $\mathbf{3 . 8 3 1 7 0 5 9 7}$ & $\mathbf{3 . 8 3 1 7 0 5 9 9}$ \\
2,1 & $\mathbf{1 . 7 4 1 4 3 5 9 7}$ & $\mathbf{1 . 7 4 1 4 3 5 9} 3$ & $\mathbf{2 . 7 0 9 4 2 5 2} 8$ & $\mathbf{2 . 7 0 9 4 2 5 2 9}$ \\
2,2 & $\mathbf{3 . 4 8 2 8 7 1 9} 4$ & $\mathbf{3 . 4 8 2 8 7 1 9 9}$ & $\mathbf{2 . 7 0 9 4 2 5} 28$ & $\mathbf{2 . 7 0 9 4 2 5 3 0}$ \\
2,4 & $\mathbf{6 . 9 6 5 7 4 3 8 7}$ & $\mathbf{6 . 9 6 5 7 4 3 8 5}$ & $\mathbf{2 . 7 0 9 4 2 5 2} 8$ & $\mathbf{2 . 7 0 9 4 2 5 2 9}$ \\
\hline
\end{tabular}

Table 3: Comparison between the exact values of $M$ and $y_{0}$ with those obtained numerically, for $N=2, q=1$ and $p=0$ 


\section{Conclusions and Future Work}

This work continues the investigation in [4] and [5] where singular boundary value problems were studied on limited and non limited domains. Here, we had to adapt the numerical algorithms introduced before to the case of free boundary problems. In the future, we are planning to extend all these results to the equation $\triangle_{m} y+$ $f(y)=0$, where $\triangle_{m}$ is the degenerate laplacian operator (with $m \neq 2$ ).

\section{Acknowledgements}

Financial support from FCT is acknowledged, through project POCTI/MAT/45700/ 2002.

\section{References}

[1] B. Franchi, E. lanconelli, J. Serrin, Existence and uniqueness of nonnegative solutions of quasilinear equations in $\mathbb{R}^{n}$, Advances in Math., 118 (1996), 177243.

[2] H.G. Kaper, M.K. Kwong, Free boundary problems for Emden-Fowler equations, Diff. Int. Eqs, 3 (1990), 353-362.

[3] N.B. Konyukhova, Singular Cauchy problems for systems of ordinary differential equations, USSR Comput. Maths. Math. Phys., 23 (1983), 72-82.

[4] P.M. Lima, N.B. Konyukhova, A.I. Sukov, N.V. Chemetov, AnalyticalNumerical Investigation of Bubble-Type Solutions of Nonlinear Singular Problems, J. Comput. Appl. Math., 189 (2006), 260-273.

[5] P. Lima, L. Morgado, Numerical approximation of singular boundary value problems for a nonlinear differential equation, submited to the Proceedings of Equadiff 11, 2005.

[6] G. Miller, V. Faber, A.B. White Jr., Finding plasma equilibria with magnetic islands, J. Computation Physics, 79 (1998), 417-435. 